\title{
El perdón en adolescentes y jóvenes: una propuesta para su medición en Argentina
}

\author{
Lucas Marcelo Rodríguez ${ }^{1}$, Maria Candela Tortul², María Soledad Menghi ${ }^{3}$ y José \\ Eduardo Moreno ${ }^{4}$
}

\section{RESUMEN}

El perdón es un proceso que implica un cambio en las emociones y las actitudes hacia la persona que ha realizado una ofensa. En este informe se presentan dos estudios. El Estudio 1 tiene como objetivo evaluar la validez de constructo y la confiabilidad de la adaptación de la Escala de Perdón de Mark Rye (1998; Rye et al., 2001) a la lengua española en jóvenes. La muestra estuvo compuesta por 323 jóvenes estudiantes universitarios de ambos sexos, de entre 18 y 28 años $(M=21.18, D E=2.35)$. Se obtuvo evidencia que valida la misma estructura factorial en la adaptación al español que la escala original, si bien se redujo el número de ítems a 10, 5 para cada una de las dos dimensiones (presencia de actitudes positivas y ausencia de actitudes negativas). Se obtuvieron medidas de consistencias interna satisfactorias. El Estudio 2 tiene como objetivo evaluar la estructura factorial de la Escala de Perdón surgida en el Estudio 1 y realizar estudios de validez convergente con Probabilidad de Perdón y con Emociones Positivas en población de adolescentes. La muestra estuvo compuesta por 401 adolescentes de ambos sexos, de entre 13 y 17 años $(M=15.43, D E=1.2)$. Se realizó un análisis factorial confirmatorio para validar la estructura en adolescentes con buenos resultados psicométricos. Ambas dimensiones de la Escala de Perdón correlacionaron positivamente con la Probabilidad de Perdón. Asimismo, se observaron asociaciones significativas con Emociones Positivas. De este modo, el Optimismo y la Serenidad correlacionaron positivamente tanto con presencia de actitudes positivas como con ausencia de negativas. Además la dimensión presencia de actitudes positivas correlacionó positivamente con Interés-entusiasmo, Alegría y sentido del humor y Gratitud. Se reflexionan los resultados hallados a la luz de la teoría acerca de las emociones positivas. Palabras clave: perdón, emociones positivas, adolescencia, juventud, escalas psicométricas.

\section{Forgiveness in Adolescents and Young People. A Proposal for its Measurement in Argentina}

\begin{abstract}
Forgiveness is a process that involves a change in the emotions and the attitudes towards the person who has made an offense. Two studies are presented in this report. The first study aimed to evaluate the construct validity and reliability of the Spanish translation and youth adaptation of Mark Rye's Forgiveness Scale (1998, Rye et al., 2001). The sample comprised 323 young university students from both sexes, between 18 and 28 years old $(M=21.28, S D=2.35)$. The evidence obtained has the same factorial structure in the scale adaptation to Spanish as in the original one. Although the number of items was reduced to 10, five for each dimension (presence of positive attitudes and absence of negative attitudes). The second study aimed to carry out a confirmatory analysis of the Forgiveness Scale adaptation, as it resulted in the first study; as well as to develop

1, 2, 3 y 4 Centro Interdisciplinario de Investigaciones en Psicología Matemática y Experimental (CIIPMECONICET), Universidad Católica Argentina, Facultad Teresa de Ávila, Sede Paraná, Argentina; lucasmarcelorodriguez@gmail.com
\end{abstract}


convergent validity studies with the Forgiveness Probability and Positive Emotions Scales, in adolescents. The sample consisted of 401 adolescents from both sexes, between 13 and 17 years old $(M=15.43, S D=1.2)$. A confirmatory factorial analysis was performed to validate the factorial structure of the scale in adolescents, with good psychometric results. Besides, both dimensions of the Forgiveness Scale correlated positively with the Probability of Forgiveness Scale. Likewise, Optimism and Serenity correlated positively with both the presence of positive and the absence of negative attitudes. In addition, the positive presence dimension positively correlated with Interest, Happiness and Sense of Humor, Enthusiasm and Gratitude. The results, found in the light of the theory of the positive emotions, are analyzed.

Keywords: forgiveness, positive emotions, adolescence, youth, psychometric scales.

El perdón es un proceso, o el resultado de este, que implica un cambio en las emociones y las actitudes hacia la persona que ha realizado una ofensa. Este proceso es para muchos autores deliberado y voluntario (American Psychological Association, 2006). Según Enright (1999; Enright, Freedman, \& Rique, 1998; Enright \& Rique, 2000) el perdón interpersonal involucra las esferas afectiva, cognitiva y conductual del desarrollo humano. Puede ser definido como "la voluntad de abandonar el resentimiento al que uno tiene derecho, los juicios negativos, y la conducta indiferente hacia quien nos dañó injustamente, mientras que se fomentan las cualidades de compasión, generosidad e incluso amor hacia él o ella" (Enright \& Rique, 2000, p. 1).

Para este autor las ideas claves en cuanto al perdón son: la reducción de sentimientos, pensamientos y conductas negativas y el desarrollo de sentimientos, pensamientos y conductas positivas hacia el ofensor. De este modo, cuando ocurre el proceso del perdón, por un lado, la importancia de los elementos negativos generados por el ofensor en cada una de estas esferas se reduce. En la esfera afectiva, las emociones negativas como la ira, odio, tristeza, entre otras, se abandonan. Esto puede ocurrir lentamente, pero finalmente se logra perdonar específicamente a esa persona en el contexto en el cual se produjo la ofensa. En la esfera cognitiva cuando se va desarrollando el proceso del perdón, los juicios condenatorios y la planificación de una venganza desaparecen. En el dominio conductual no se lleva a cabo la venganza, si se estaba planificando. Por otra parte, durante el proceso de perdonar, los elementos positivos en lo afectivo, cognitivo y conductual aumentan su fuerza. Así, en la esfera afectiva, las emociones negativas son reemplazadas por emociones más neutrales y eventualmente emociones positivas, tal como la compasión, el ágape y el amor (Enright, 1999; Enright et al., 1998; Enright \& Rique, 2000).

Casullo (2006), investigadora argentina, considera al perdón como un proceso y lo caracteriza como un constructo psicosocial mediado por una dimensión subjetiva intrapsíquica y otra interpersonal. Se trata de un proceso complejo ya que involucra las esferas afectiva, cognitiva, motivacional y comportamental (Casullo, 2008).

A partir de las conceptualizaciones de perdón interpersonal, Rye et al. (2001) definen el perdón como la respuesta hacia un ofensor que implica dejar de lado el afecto, cogniciones y conductas negativas, para desarrollar actitudes positivas hacia el mismo. Esta relación entre los constructos de perdón interpersonal, tanto de Enright como de Rye, ha quedado en evidencia en estudios empíricos (Rye et al., 2001) al observarse la correlación positiva que 
tienen la Escala de Probabilidad de Perdón y el Inventario de Perdón de Enright (Enright \& Rique, 2000).

El perdón ha sido estudiado en los últimos años, con su evidencia de beneficios para el desarrollo socioemocional de las personas y efectos positivos sobre la salud mental y el bienestar (Castro Solano, 2010; Coyle \& Enright, 1997). Así, por ejemplo, estudios actuales han mostrado una elevada asociación positiva entre perdón y salud mental en una población de pacientes deprimidos (Abid, 2017). Otros estudios empíricos han asociado la falta de perdón con el aumento de la afectividad negativa, la disminución de las habilidades cognitivas y el desarrollo de barreras para el crecimiento psicológico y social. La contracara se ha evidenciado en que el perdón se asocia con reducción del afecto negativo. Además se asocia con la presencia de sentimientos positivos, relaciones positivas con los demás y sentido de significado y propósito en la vida; todo esto relacionado con el bienestar psicológico (Akhtar, Dolan, \& Barlow, 2017).

Cabe aclarar que en este estudio se aborda el perdón interpersonal, el cual debe distinguirse del perdón a uno mismo. Este último adquiere características diferentes y muchas veces se lo ha intentado estudiar erróneamente desde las teorizaciones del perdón interpersonal (Pietro Urzúa \& Echegoyen, 2015). En este marco de diferentes definiciones de perdón y diversos niveles de especificidad —como perdón interpersonal o a uno mismo- es que se han desarrollado una variedad de escalas de autoinforme para su medición y abordaje científico (Casullo, 2008; Regalia \& Paleari, 2014). No obstante, hay autores que remarcan la necesidad de adaptar este tipo de escalas al idioma español (Rosales Sarabia, Rivera Aragón, \& García Méndez, 2017).

En el ámbito nacional, Casullo (2005; Casullo \& Fernández Liporace, 2005) desarrolló una escala de perdón denominada CAPER. La misma evalúa el perdón teniendo en cuenta el sí mismo, los otros y las situaciones; además evalúa el impacto de creencias acerca de un poder superior y del destino en la capacidad de perdonar. También se han realizado trabajos de traducción, adaptación y validación de escalas para la población argentina, como es el caso de la adaptación de la Escala de Probabilidad de Perdón (Menghi, Rodriguez, Tortul, \& Moreno, 2017), entre otras.

Casullo (2006), en sus trabajos en la población argentina, ha puesto de manifiesto la relevancia que adquiere el perdón para las personas. Los adolescentes y jóvenes ven el perdón como importante para obtener una segunda oportunidad frente a equivocaciones. Además se han visto diferencias en las motivaciones para el perdón entre hombres y mujeres; los hombres suelen perdonar para olvidar y seguir adelante, con lo que se mejora así la convivencia social; por su parte, las mujeres perdonan para obtener el mismo beneficio en el caso en que tuviesen que ser perdonadas.

Teniendo en cuenta las teorizaciones del perdón como un proceso en el cual disminuyen afectos, cogniciones y conductas negativas hacia el ofensor y aumentan los afectos, cogniciones y conductas positivas hacia el mismo, el perdón es un constructo que debe vincularse con la emocionalidad positiva, más concretamente con las emociones positivas, y puede abordarse la relación entre ambos constructos.

Las emociones positivas son experiencias emocionales en las que predomina el placer o bienestar (Lucas, Diener, \& Larsen, 2003). Para algunos autores una emoción positiva verdadera debe añadir una interpretación 
personal positiva del suceso, es decir que no alcanza con experimentar agradabilidad sensorial, la emoción debe generar cambios subjetivos a nivel fisiológico, psicológico, conductual e incluso social (Fredrickson, 2000). Las emociones positivas son distintas de los estados de ánimo positivos, ya que las mismas tienen un objeto específico, son de corta duración y ocupan un primer plano en la conciencia, contrariamente a los estados de ánimo positivos (Fredrickson \& Cohn, 2008).

Las emociones positivas tienen efectos positivos en distintas áreas del funcionamiento psicológico (Hervás, 2009). Así, por ejemplo, impulsan el desarrollo de habilidades y comportamientos nuevos (Fredrickson, 1998), la resolución de problemas, el aumento del altruismo y la creatividad, etc. (Avia \& Vazquez, 1998, como se citó en Hervás, 2009).

Entre las principales emociones positivas pueden considerarse: a) la alegría: capacidad de reír y sonreír; b) el sentido del humor: entendido como la capacidad de divertirse a sí mismo y a los demás; c) el optimismo: emoción caracterizada por esperar que sucedan cosas buenas, mirando siempre el lado positivo de las cosas y de los problemas; d) la serenidad o tranquilidad: caracterizada por un bienestar profundo que se da al desconectarse de pensamientos inquietantes y al tomar distancia de las preocupaciones, el estrés y el miedo; e) la gratitud: emoción de aprecio por alguien o algo y buen deseo hacia esa persona o cosa, lo que hace surgir el deseo de actuar positivamente; f) el interés y el entusiasmo: emoción que motiva hacia el cambio y la novedad; abarca la activación y la orientación para mantener a la persona activamente comprometida con el mundo; y finalmente g) la satisfacción con la vida: emoción de contentamiento, paz y satisfacción, resultado de la comparación entre querer y necesitar y los logros y habilidades alcanzadas (Schmidt, 2008).

Teniendo en cuenta lo expresado teóricamente, se planteó realizar dos estudios. El Estudio 1 tiene como objetivo evaluar la validez del constructo y la confiabilidad de la adaptación de la Escala de Perdón de Rye (1998; Rye et al., 2001) a la lengua española en jóvenes. El Estudio 2 tiene como objetivo evaluar la estructura factorial de la Escala de Perdón surgida en el Estudio 1 y realizar estudios de validez convergente con la Probabilidad de Perdón y con las Emociones Positivas en adolescentes.

\section{ESTUDIO 1}

\section{METODOLOGÍA}

\section{Participantes}

Se realizó un muestreo no probabilístico, intencional. Participaron de la investigación 323 jóvenes estudiantes universitarios de ambos sexos, de la ciudad de Paraná (Entre Ríos, Argentina), de entre 18 y 28 años; 48 varones y 275 mujeres. La media de edad fue de $21.18(D E=2.35)$. Los mismos cursaban las siguientes carreras: Psicología, Psicopedagogía, Abogacía, Relaciones Internacionales y Ciencias Politicas. Los alumnos pertenecen a una universidad privada, en donde la mayoria proviene de familias de un nivel socioeconómico medio o alto. 


\section{Materiales}

Los instrumentos utilizados para la recolección de datos fueron los siguientes: un cuestionario ad hoc para la obtención de datos sociodemográficos y la Escala de Perdón (The Forgiveness Scale) de Rye (1998; Rye et al., 2001). La Escala de Perdón consta de 15 items en dos dimensiones: la dimensión de ausencia de sentimientos, pensamientos y conductas negativos hacia el ofensor (10 items) y la dimensión de presencia de sentimientos, pensamientos y conductas positivos hacia el ofensor (5 items). Se responde mediante una escala tipo Likert de 5 opciones a saber: 1. Totalmente en desacuerdo, 2. En desacuerdo, 3. Ni en desacuerdo ni de acuerdo, 4. De acuerdo, 5. Totalmente de acuerdo.

Se realizó una adaptación a la lengua española (para la población argentina) de la escala original de Rye et al. (2001). Para ello en primer lugar se obtuvieron traducciones del inglés al español de tres personas calificadas para tal fin. Se realizó una traducción funcional del test. Luego se llevó a cabo una revisión de la adaptación con expertos en la temática del perdón. Se tuvieron en cuenta los pasos propuestos por Hambleton y Patsule (1999) para realizar el procedimiento.

\section{Procedimiento}

Se realizaron entrevistas con las autoridades universitarias a fin de solicitar la autorización para efectuar las evaluaciones. Una vez obtenidos los permisos correspondientes se comenzó con las evaluaciones, las cuales tuvieron lugar en grupos dentro de las aulas debido a que los instrumentos eran autoadministrables. En cada toma se encontraron presentes miembros del equipo investigador para controlar y responder, en caso en que existiese, alguna duda en cuanto a la tarea a realizar. Previo a la participación cada sujeto firmó el correspondiente consentimiento informado.

Para los procedimientos estadísticos se utilizó el análisis factorial exploratorio (AFE) mediante el método de componentes principales y se utilizó el índice alfa de Cronbach. Dichos análisis se realizaron con el paquete estadístico para ciencias sociales (SPSS).

\section{RESULTADOS}

Para evaluar la validez de constructo se hizo un análisis factorial exploratorio (AFE) mediante el método de componentes principales y la rotación ortogonal (método Varimax), con los 15 items que componen la Escala de Perdón de Rye (1998; Rye et al., 2001). Se fijó el número de factores en dos según lo esperado teóricamente. Previamente al mismo se calculó el índice de adecuación muestral de Kaiser-Meyer-Oklin $(K M O=.80)$ y la prueba de esfericidad de Bartlett $\left(X^{2}=1070.38 p=.000\right)$, los cuales evidenciaron que era pertinente realizar un AFE. A partir de los resultados del AFE las dos dimensiones explicaron el $41.07 \%$ de la variancia. El primer factor explicó el $21.12 \%$ de la variancia y el segundo explicó el $19.95 \%$. En la tabla 1 pueden apreciarse las cargas factoriales de cada ítem de la Escala de Perdón. 
Tabla 1.

Primer Análisis Factorial Exploratorio de la Escala de Perdón (15 ítems).

\begin{tabular}{|c|c|c|c|}
\hline \multirow{2}{*}{\multicolumn{2}{|c|}{ Ítems }} & \multicolumn{2}{|c|}{ Componentes } \\
\hline & & $\begin{array}{l}\text { Presencia de } \\
\text { actitudes } \\
\text { Positivas }\end{array}$ & $\begin{array}{l}\text { Ausencia } \\
\text { de actitudes } \\
\text { Negativas }\end{array}$ \\
\hline & $\begin{array}{l}\text { No puedo dejar de pensar, evitar recordar, en cómo esta persona me } \\
\text { ofendió.* }\end{array}$ & .28 & .47 \\
\hline \multirow{2}{*}{$\begin{array}{l}2 \\
3\end{array}$} & \multirow{2}{*}{$\begin{array}{l}\text { Deseo que le sucedan cosas buenas a la persona que me dañó. } \\
\text { Paso mucho tiempo pensando en cómo vengarme de la persona que me } \\
\text { ofendió.* }\end{array}$} & .62 & .06 \\
\hline & & .32 & .42 \\
\hline 4 & Estoy resentido con la persona que me dañó, sigo con la bronca. ${ }^{*}$ & .38 & .42 \\
\hline 5 & Evito lugar o personas que me recuerdan a quien me ofendió. ${ }^{*}$ & -.01 & .57 \\
\hline 6 & Rezo por la persona que me dañó. & .76 & -.07 \\
\hline 7 & Si me encontrara con la persona que me dañó me sentiría en paz. & .58 & .08 \\
\hline 8 & El daño que me hizo esta persona me impide disfrutar de la vida. ${ }^{*}$ & -.04 & .74 \\
\hline 9 & He podido librarme del enojo o la bronca hacia la persona que me ofendió. & .44 & .29 \\
\hline 10 & Me deprimo cuando pienso cómo me maltrató esa persona.* & -.06 & .69 \\
\hline 11 & $\begin{array}{l}\text { Creo que ya han sanado muchas de las heridas provocadas por esa } \\
\text { persona. }\end{array}$ & .27 & .35 \\
\hline 12 & Siento odio cada vez que pienso en la persona que me dañó.* & .43 & .54 \\
\hline 13 & Tengo compasión por la persona que me dañó. & .72 & .01 \\
\hline 14 & $\begin{array}{l}\text { Creo que mi vida está arruinada a causa de los agravios recibidos de esa } \\
\text { persona.* }\end{array}$ & -.03 & .71 \\
\hline \multirow[t]{2}{*}{15} & $\begin{array}{l}\text { Espero que la persona que me dañó sea bien tratada por los demás en el } \\
\text { futuro. }\end{array}$ & .72 & .05 \\
\hline & $\%$ de variancia explicada por cada factor. & 21.12 & 19.95 \\
\hline
\end{tabular}

* Ítem de puntuación invertida

$K M O=.80, X^{2}=1070.38, p=.000$

$n=323$

A partir de los resultados del AFE obtenidos con la Escala de Perdón de 15 ítems, se evaluó la pertinencia de 5 items. Los ítems 3, 4, 11 y 12 cargaron en ambos factores, por lo cual se decidió sacarlos de la escala. El ítem 9 cargó en presencia de actitudes positivas, cuando teóricamente se esperaba que cargara en ausencia de negativas, por tal motivo también se decidió quitarlo de la escala.

Se llevó a cabo un segundo AFE con los 10 ítems resultantes del primer AFE. Se fijó el número de factores en dos según lo esperado teóricamente. Previamente al mismo se calculó el índice de adecuación muestral de KaiserMeyer-Oklin $(K M O=.76)$ y la prueba de esfericidad de Bartlett $\left(X^{2}=662.49 p\right.$ $=.000$ ), los cuales revelaron que era pertinente realizar un AFE. A partir de los resultados del AFE las dos dimensiones explicaron el $49.72 \%$ de la variancia. El primer factor explicó el $26.58 \%$ de la variancia y el segundo explicó el $23.14 \%$. En la tabla 2 pueden apreciarse las cargas factoriales de cada ítem de la Escala de Perdón. 
Tabla 2.

Segundo Análisis Factorial Exploratorio de la Escala de Perdón (10 ítems)

\begin{tabular}{|c|c|c|c|}
\hline & \multirow[b]{2}{*}{ Ítems } & \multicolumn{2}{|c|}{ Componentes } \\
\hline & & $\begin{array}{c}\text { Presencia de } \\
\text { actitudes } \\
\text { Positivas }\end{array}$ & $\begin{array}{c}\text { Ausencia } \\
\text { de actitudes } \\
\text { Negativas }\end{array}$ \\
\hline 1 & $\begin{array}{l}\text { No puedo dejar de pensar, evitar recordar, en cómo esta persona me } \\
\text { ofendió.* }\end{array}$ & .31 & .48 \\
\hline 2 & Deseo que le sucedan cosas buenas a la persona que me dañó. & .66 & .08 \\
\hline 5 & Evito lugar o personas que me recuerdan a quien me ofendió. ${ }^{*}$ & .01 & .61 \\
\hline 6 & Rezo por la persona que me dañó. & .78 & -.07 \\
\hline 7 & Si me encontrara con la persona que me dañó me sentiría en paz. & .61 & .10 \\
\hline 8 & El daño que me hizo esta persona me impide disfrutar de la vida. ${ }^{*}$ & .02 & .79 \\
\hline 10 & Me deprimo cuando pienso cómo me maltrató esa persona.* & -.03 & .72 \\
\hline 13 & Tengo compasión por la persona que me dañó. & .76 & .05 \\
\hline 14 & $\begin{array}{l}\text { Creo que mi vida está arruinada a causa de los agravios recibidos de esa } \\
\text { persona. }\end{array}$ & .03 & .73 \\
\hline \multirow[t]{2}{*}{15} & $\begin{array}{l}\text { Espero que la persona que me dañó sea bien tratada por los demás en el } \\
\text { futuro. }\end{array}$ & .72 & .02 \\
\hline & $\%$ de variancia explicada por cada factor. & 26.58 & 23.14 \\
\hline
\end{tabular}

Luego del segundo AFE, se realizó el análisis de consistencia interna de la escala a partir del índice alfa de Cronbach. El alfa para la totalidad de la escala (10 items) fue de .70. El alfa para la escala de presencia de actitudes positivas de 5 ítems fue de .77 , en tanto el alfa para la escala de ausencia de actitudes negativas de 5 items fue de .67 .

\section{COMENTARIOS}

En relación al Estudio 1, el objetivo era evaluar la validez de constructo y la confiabilidad de la adaptación de la Escala de Perdón de Rye (1998; Rye et al., 2001) a la lengua española en jóvenes.

Se efectuaron dos AFE para evaluar la validez de constructo. Previamente se calculó el índice de adecuación muestral de Kaiser-Meyer-Oklin. Esta medida (KMO) expresa cuán grande es la correlación entre las variables que se miden; si la correlación es suficientemente grande se considera que la matriz es adecuada para su factorización, ya que ofrece resultados estables y replicables (Lloret Segura, Ferreres Traver, Hernández Baeza, \& Tomás Marco, 2014). Para Kaiser (1970), valores menores a .50 indican matrices inadecuadas para análisis factorial; valores entre .60 y .69 son mediocres, mientras que valores de .80 o superiores son satisfactorios para realizar análisis factorial. Otros autores indican que valores superiores a .70 son satisfactorios para realizar análisis factorial (Lloret Segura et al., 2014). En el presente estudio tanto el primer AFE como el segundo presentaron índices KMO adecuados para realizar AFE.

Para una estructura factorial simple existen ciertos requisitos: que la variancia esté uniformemente distribuida respecto de los factores, que cada variable solo cargue en un factor, que los pesos de los factores estén próximos a 1 o 0 y que los factores sean unipolares (Norman \& Streiner, 1996). En el caso del primer AFE realizado, estos requisitos no se cumplian en su totalidad, debido a que los ítems 3 (Paso mucho tiempo pensando en cómo vengarme de la 
persona que me ofendió, invertido), 4 (Estoy resentido con la persona que me dañó, sigo con la bronca, invertido), 11 (Creo que ya han sanado muchas de las heridas provocadas por esa persona) y 12 (Siento odio cada vez que pienso en la persona que me dañó, invertido) cargaron tanto en presencia de actitudes positivas como en ausencia de negativas. Además, el ítem 9 (He podido librarme del enojo o la bronca hacia la persona que me ofendió) si bien presentaba una carga factorial compleja, pesaba más en el factor presencia de actitudes positivas, cuando teóricamente se esperaba que perteneciera a ausencia de negativas. Estas dificultades con items complejos fueron halladas también en algunos ítems en la escala original. Así por ejemplo los ítems 4 y 12 cargaron en ambos factores en la versión del autor (Rye et al., 2001). A partir de estos resultados se decidió eliminar los 5 items mencionados, los cuales eran de la dimensión ausencia de actitudes negativas. De esta manera quedaria un instrumento de dos dimensiones con 5 items por cada dimensión, ya que el factor presencia de positivas no ha sufrido modificaciones en relación a la escala original.

A partir de esto se realizó un segundo AFE con los 10 ítems, y se obtuvo una estructura factorial simple, con las características antes mencionadas (Norman \& Streiner, 1996).

En cuanto a la confiabilidad del instrumento, se calculó el índice alfa de Cronbach. Dicho índice se considera una buena estimación de consistencia interna cuando su valor fluctúa entre .70 y .80 (Kaplan \& Saccuzzo, 2006). Valores algo más bajos, alrededor de .60 , pueden ser indicadores de buena consistencia interna en escalas con escaso número de items, pero buena evidencia de validez (Loewenthal, 2001). Estos parámetros dejan en evidencia que los resultados obtenidos son una prueba de buena consistencia interna para la escala total (10 items) y para la dimensión presencia de positivo. No así la dimensión ausencia de negativo que ha presentado una confiabilidad más baja. Para la escala original de Rye et al. (2001) los alfas informados fueron .85 para presencia de positivas y .86 para ausencia de negativas. También estudios recientes han mostrado buena consistencia interna (Boonyarit, 2017). Si bien los coeficientes alfa del presente estudio son más bajos que la escala original, cabe aclarar que la presente adaptación tiene menor número de items; sin embargo, los coeficientes alfa muestran una buena consistencia interna siguiendo el criterio de los autores mencionados.

\section{ESTUDIO 2}

\section{METODOLOGÍA}

\section{Participantes}

Se realizó un muestreo no probabilístico, intencional. Participaron de la investigación 401 adolescentes de ambos sexos, 182 varones y 219 mujeres de entre 13 y 17 años. La media de edad fue de $15.43(D E=1.2)$. Todos pertenecen a escuelas de nivel medio de la provincia de Entre Ríos, Argentina. Las instituciones educativas a la que asisten los alumnos que participaron del estudio, tanto las públicas como las de gestión privada de Paraná (provincia de Entre Ríos) según su ubicación geográfica se encuentran en zonas dentro de los límites urbanos, ubicadas en barrios de estrato medio, y medio alto, por lo cual se estima que los sujetos pertenecen a niveles socioeconómicos medios. 
Cabe aclarar que para estudiar la validez convergente con Emociones Positivas se tomó una submuestra de la muestra citada en el párrafo anterior. Esto se debió a que solo pudo evaluarse una escuela con el cuestionario de Emociones Positivas. Las características de la misma fueron las siguientes: 135 adolescentes (61 varones y 74 mujeres); en cuanto a la edad, $M=14.56$, $D E=1.14$.

\section{Materiales}

Los instrumentos utilizados para la recolección de datos fueron los siguientes: un cuestionario ad hoc para la obtención de datos sociodemográficos, la Escala de Perdón de Rye (1998; Rye et al., 2001) (ver materiales del Estudio 1), y el Cuestionario de Emociones Positivas de Schmidt (2008), en su versión abreviada (Rodriguez, Oñate, \& Mesurado, 2017) de 31 items. Dicho instrumento comprende una puntuación tipo Lickert que va desde muy en desacuerdo (1) a muy de acuerdo (4). Mide las siguientes emociones positivas: Alegría y sentido del humor, Optimismo, Serenidad, Gratitud, Interés-entusiasmo y Satisfacción con la vida. Los alfas de Cronbach informados para las diversas subescalas fueron los siguientes: Alegría y sentido del humor: .88, Optimismo: .72 Serenidad: .85, Gratitud: .68, Interésentusiasmo: .68 y Satisfacción con la vida: .65 (Rodriguez et al., 2017).

Además se utilizó la Escala de Probabilidad de Perdón de Rye (1998; Rye et al., 2001), la cual mide la probabilidad de perdón en diferentes escenarios y situaciones posibles de agravio. El mismo consta de 10 situaciones en las que se produce una ofensa o agravio. En la situación hipotética planteada en cada item, la persona que responde la escala es agraviada y se le pregunta por la probabilidad de que perdone. La modalidad de respuesta es mediante una escala tipo Likert de 5 opciones a saber: 1 (nada probable), 2 (poco probable), 3 (algo probable), 4 (bastante probable) y 5 (totalmente probable). Se utilizó la adaptación al español de Menghi et al. (2017). La consistencia interna de la adaptación al español fue de .76.

\section{Procedimiento}

Se realizó una entrevista con autoridades de las escuelas de nivel medio a fin de obtener la autorización para realizar las evaluaciones en los establecimientos educativos. Una vez obtenidos los permisos correspondientes se enviaron notas a los padres o tutores a cargo de los alumnos con el consentimiento informado. Las evaluaciones se realizaron en grupos dentro de las aulas ya que los instrumentos eran autoadministrables. En las mismas participaron por lo menos dos investigadores del proyecto para controlar y responder a las dudas que pudiesen surgir por parte de los evaluados. Se administraron las escalas a aquellos adolescentes que estuvieron autorizados, es decir que presentaron el consentimiento de sus padres o tutores a cargo y que además aceptaron voluntariamente participar en la investigación.

Para los procedimientos estadísticos se utilizó análisis factorial confirmatorio (AFC) con el método de estimación de parámetros por máxima verosimilitud, indice alfa de Cronbach y correlaciones de Pearson. Dichos análisis se realizaron con los paquetes estadísticos SPSS y AMOS. 


\section{RESULTADOS}

Para poner a prueba el modelo de 10 ítems de la Escala de Perdón surgido del segundo AFE del Estudio 1, se llevó a cabo un análisis factorial confirmatorio (AFC) con el método de estimación de parámetros por máxima verosimilitud. Para evaluar el ajuste del modelo se tuvieron en cuenta el índice comparativo de ajuste $(C F I)$, el índice de bondad de ajuste (GFI) y el indice ajustado de bondad de ajuste $(A G F I$. Se calcularon como medidas del error el Root Mean Square Residual $(R M R)$ y el promedio de los residuales estandarizados al cuadrado (RMSEA). Los resultados del ajuste del modelo fueron los siguientes: $\chi^{2}(34)=67.61, p=.001, \chi^{2} / g l=1.99, C F I=.94, G F I=$ $.97, A G F I=.95, R M R=.072$ y $R M S E A=.05$. Dichos resultados indicaron un buen ajuste del modelo propuesto. En la figura 1 puede observarse el modelo de medición y las cargas factoriales estandarizadas de la Escala de Perdón.

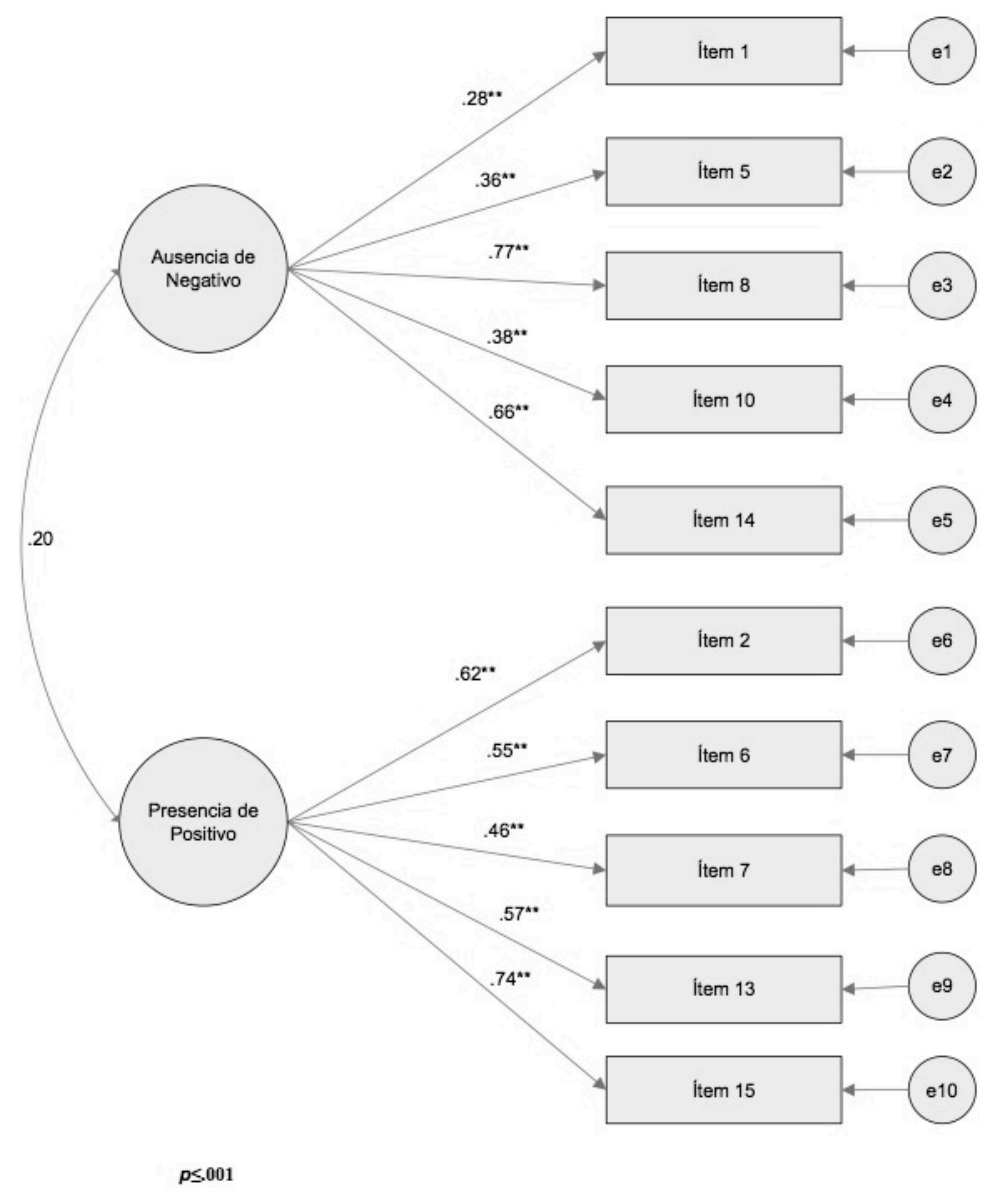

Figura 1. Modelo de medición de la Escala de Perdón

Se realizó un análisis de consistencia interna de la escala a partir del indice alfa de Cronbach. El alfa para la escala de Ausencia de Actitudes Negativas fue de .59 mientras que el alfa para la escala de Presencia de Actitudes Positivas fue de .72.

Para evaluar la validez convergente se realizaron correlaciones de Pearson con las dos dimensiones de la Escala de Perdón y la Escala de Probabilidad de Perdón. En la tabla 3 pueden apreciarse las correlaciones. 
Tabla 3.

Correlaciones de Pearson de la Escala de Perdón y la Escala de Probabilidad de Perdón.

\begin{tabular}{lccc}
\hline & $\mathbf{1}$ & $\mathbf{2}$ & $\mathbf{3}$ \\
\cline { 2 - 4 } 1 Presencia de Actitudes Positivas & 1 & & \\
2 Ausencia de Actitudes Negativas & $.11^{*}$ & 1 & \\
3 Escala de Probabilidad de Perdón & $.32^{* *}$ & $.14^{\star *}$ & 1 \\
\hline${ }^{*}$. La correlación es significativa en el nivel .01. & & & \\
*. La correlación es significativa en el nivel .05. \\
$n=400$
\end{tabular}

Además, se realizaron correlaciones de Pearson con las dos dimensiones de la Escala de Perdón y las emociones positivas Interés-Entusiasmo, Serenidad, Satisfacción con la Vida, Alegria y Sentido del Humor, Optimismo y Gratitud. En la tabla 4 pueden apreciarse las correlaciones.

Tabla 4.

Correlaciones de Pearson de la Escala de Perdón y las Emociones Positivas.

\begin{tabular}{lcccccccc}
\hline & 1 & 2 & 3 & 4 & 5 & 6 & 7 & 8 \\
\hline 1 Presencia de Positivo & 1 & & & & & & & \\
2 Ausencia de Negativo & .07 & 1 & & & & & & \\
3 Interés - Entusiasmo & $.26^{* *}$ & -.08 & 1 & & & & & \\
4 Serenidad & $.27^{* *}$ & $.18^{*}$ & .10 & 1 & & & & \\
5 Satisfacción con la Vida & -.04 & .11 & .08 & $.21^{*}$ & 1 & & & \\
6 Alegría y Sentido del Humor & $.25^{* *}$ & .08 & $.44^{* *}$ & .04 & .07 & 1 & & \\
7 Optimismo & $.36^{* *}$ & $.23^{* *}$ & $.28^{* *}$ & $.32^{* *}$ & -.00 & $.34^{* *}$ & 1 & \\
8 Gratitud & $.19^{*}$ & .12 & .11 & $.17^{*}$ & .07 & $.27^{* *}$ & $.19^{*}$ & 1 \\
\hline$*$ La correlación es significativa en el nivel .01. & & & & & & & \\
*. La correlación es significativa en el nivel .05. & & & & & & & \\
$n=135$ & & & & & & & &
\end{tabular}

\section{COMENTARIOS}

En relación al Estudio 2, el objetivo fue evaluar la estructura factorial de la Escala de Perdón de 10 ítems surgida en el segundo AFE citado y realizar estudios de validez convergente con Probabilidad de Perdón y Emociones Positivas en la población de adolescentes.

Se llevó a cabo un análisis factorial confirmatorio. Los resultados del mismo indicaron un buen ajuste del modelo teórico propuesto a los datos empíricos. Esto puede afirmarse debido a que el índice comparativo de ajuste $(C F I)$, el indice de bondad de ajuste $(G F I)$ y el indice ajustado de bondad de ajuste $(A G F I)$ fueron superiores a .90; asimismo el $\chi^{2} / g l$ fue inferior a 2 y las medidas del error RMR y RMSEA estuvieron alrededor de 60 (Cupani, 2012; Kline, 1998). De esta manera se evalúa positivamente la estructura de la Escala de Perdón, y se mantienen las dos dimensiones originales del autor (Rye et al., 2001) a saber: presencia de actitudes positivas y ausencia de negativas, con la disminución del número de ítems justificada en el primer estudio.

En cuanto a la validez convergente, se realizaron correlaciones de Pearson de las dimensiones de la Escala de Perdón con la Escala de Probabilidad de Perdón. Se hallaron correlaciones positivas significativas entre la probabilidad de perdón y la dimensión ausencia de actitudes negativas, así como la probabilidad de perdón y la dimensión presencia de actitudes positivas, en donde la última fue la más elevada. Es teóricamente clara la 
relación entre las escalas, ya que se espera que las personas que presentan ausencia de pensamientos, sentimientos y conductas negativas hacia el ofensor, así como presencia de pensamientos, sentimientos y conductas positivas hacia el ofensor, presenten mayores niveles de probabilidad de perdón en diferentes escenarios y situaciones posibles de agravio. Estos resultados son coincidentes con los encontrados por Rye et al. (2001), quienes hallaron que las correlaciones entre la dimensión presencia de actitudes positivas y probabilidad de perdón eran más elevadas que las correlaciones entre ausencia de negativas y probabilidad de perdón.

Además, se realizaron correlaciones de Pearson de ambas dimensión de la Escala de Perdón con algunas emociones positivas, a saber: interés y entusiasmo, serenidad, satisfacción con la vida, alegría y sentido del humor, optimismo y gratitud. Se obtuvieron correlaciones positivas significativas entre la dimensión presencia de actitudes positivas y las siguientes emociones positivas: optimismo, tranquilidad, interés y entusiasmo, alegría y sentido del humor, y gratitud. Asimismo se obtuvieron correlaciones positivas significativas entre la dimensión ausencia de negativas y las emociones optimismo y serenidad.

La dimensión presencia de actitudes positivas mide la presencia de sentimientos, pensamientos y conductas positivas hacia el ofensor (Rye, 1998; Rye et al., 2001), por tanto puede afirmarse que dicha dimensión mide cuestiones positivas en la esfera afectiva. Por su parte las emociones positivas son aquellas en las que predomina el placer o bienestar, que añaden una interpretación positiva del suceso (Fredrickson, 2000; Lucas et al., 2003). Además, las emociones positivas tienen efectos positivos en diversas áreas del funcionamiento psíquico, y promueven entre otras cosas la resolución de problemas (Hervás, 2009). En el presente estudio se observó que casi la totalidad de las emociones positivas medidas correlacionaron positivamente con presencia de actitudes positivas, lo cual puede explicarse por la emocionalidad positiva de ambos constructos (presencia de positivo y emociones positivas). Además el perdón puede entenderse como parte integrante de la resolución de problemas o conflictos, lo cual puede ser generado por la presencia de emociones positivas.

El optimismo como emoción positiva está caracterizada por la espera de que sucedan cosas buenas (Schmidt, 2008). Se espera que una visión optimista de una relación y de uno mismo influya en la capacidad de perdonar. La tendencia a ver la vida con optimismo puede ayudar a que la persona realice atribuciones menos permanentes y perjudiciales hacia el ofensor. Esta emoción además puede ayudar a que la persona vea la relación con su ofensor con expectativas positivas. Se espera que el optimismo ayude a la persona ofendida a realizar un afrontamiento positivo y lograr la resolución de conflictos, lo que facilitaría el perdón (Chang, 1998; Worthington \& Wade, 1999). Esto explica la correlación positiva hallada entre el optimismo y ambas dimensiones de la Escala de Perdón (presencia de positivo y ausencia de negativo).

Por su parte, la serenidad está caracterizada por un bienestar profundo que se da al desconectarse de pensamientos inquietantes y tomar distancia de las preocupaciones, el estrés y el miedo (Schmidt, 2008). Dicha emoción también correlacionó positivamente con ambas dimensiones de la Escala de Perdón. No se han encontrado a la fecha estudios empíricos que relacionen estos constructos (perdón y serenidad). Puede hipotetizarse que aquella 
persona que logra perdonar a sus ofensores manifiesta mayor tranquilidad en su vida como consecuencia de este proceso de perdón.

\section{CONCLUSIONES GENERALES, LIMITACIONES Y FUTUROS ESTUDIOS}

El presente estudio es un valioso aporte metodológico para la medición del perdón en población de adolescentes y jóvenes, y aporta un instrumento con sólidas propiedades psicométricas.

Entre las limitaciones del mismo debe mencionarse que las correlaciones entre Perdón y Emociones Positivas se realizaron con una pequeña parte de la muestra. Otra limitación de la presente adaptación es la baja consistencia interna de la subescala de ausencia de actitudes negativas hacia el ofensor, lo cual requiere una profundización de sus causas. En futuros estudios sería conveniente analizar la consistencia interna de esta subescala, teniendo en cuenta que en esta investigación ya se ha reducido la misma de 10 a 5 items y que los niveles de alfa de Cronbach no mejoraron con la opción de eliminación de algún ítem.

Futuras líneas de investigación deberian indagar en las diferencias que pueden observarse en el perdón en los diversos estratos sociales a los que pertenecen los participantes, y en el caso de trabajar con niños y adolescentes, tener en cuenta el nivel educativo promedio de los padres como variables de control y contextualización (ejemplo nivel educacional, empleo, profesión, etc.).

Futuros estudios deberian profundizar la relación del Perdón con las Emociones Positivas, ; pueden evaluarse la relación de cada emoción con el Perdón. Además deberían diseñarse estudios para dar cuenta de la relación causal de dichos fenómenos; es decir que indiquen si las Emociones Positivas son consecuencia de un proceso de perdón o si la puesta en marcha de un proceso de Perdón se debe a que la persona previamente vivenciaba Emociones Positivas.

\section{REFERENCIAS}

Abid, M. (2017). Forgiveness: As Attaining Mental Health among Depressed Patients. Journal Psychology and Clinical Psychiatry, 7(4), 00444. https://doi.org/10.15406/jpcpy.2017.07.00444

Akhtar, S., Donal, A., \& Barlow, J. (2017). Understanding the Relationship Between State Forgiveness and Psychological Wellbeing: A Qualitative Study. Journal of Religion and Health, 56, 450-463. https://doi.org/10.1007/s10943-016-0188-9

American Psychological Association (2006). Forgiveness: A sampling of research results. Retrieved from http://www.apa.org/international/resources/forgiveness.pdf

Boonyarit, I. (2017). Assessing Forgiveness in Interpersonal Conflict among Thai Emerging Adults: The Peer Forgiveness Scale. International Journal of Behavioral Science, 12(2), 1-18.

Castro Solano, A. (2010). Fundamentos de Psicología Positiva. Buenos Aires. Paidós.

Casullo, M. (2005). La capacidad para perdonar desdeuna perspectiva psicológica. Revista de Psicología de la PUCP, 23(1), 39-64.

Casullo, M. M. (2006). Las razones para perdonar: Concepciones populares o teorias implícitas. Psicodebate, 7, 9-20. https://doi.org/10.18682/pd.v7i0.424

Casullo, M. M. (2008). Prácticas en Psicología Positiva. Buenos Aires. Lugar Editorial.

Casullo, M. \& Fernández-Liporace, M. (2005). Evaluaciónde la capacidad de perdonar. Desarrollo y validación de una escala. Acta Psiquiátrica y Psicológica de América Latina, 51(1), 14-20.

Chang, E. C. (1998). Dispositional optimism and primary and secondary appraisal of a stressor: Controlling for confounding influences and relations to coping and psychological and physical adjustment Journal of Personality and Social Psychology, 74, 1109-1120. https://doi.org/10.1037/0022-3514.74.4.1109

Coyle, C. T. \& Enright, R. D. (1997). Forgiveness as an intervention goal with post-abortion men. Journal of Consulting and Clinical Psychology, 65, 1042-1046. https://doi.org/10.1037/0022-006X.65.6.1042

Cupani, M. (2012). Análisis de Ecuaciones Estructurales: conceptos, etapas de desarrollo y un modelo de aplicación. Revista tesis, 1, 186-199.

Enright, R. D. \& Rique, J. (2000). The Enright Forgiveness Inventory. Manual, Instrument and Scoring Guide. 
CA: Mind Garden.

Enright, R. D., Freedman, S. R., \& Rique, J. (1998). The psychology of interpersonal forgiveness. En R. Enright \& J. North (Eds.), Exploring Forgiveness. Madison WI: University of Wisconsin Press.

Enright, R. D. (1999). Forgiveness. Encyclopedia of emotions and behavior. New York: Macmillan.

Fredrickson, B. L. (1998). What good are positive emotions? Review of General Psychology, 2, 300-319. https://doi.org/10.1037/1089-2680.2.3.300

Fredrickson, B. L. (2000). Cultivating positive emotions to optimize health and well-being. Prevention \& Treatment, 3(1), 1a. https://doi.org/10.1037/1522-3736.3.1.31a

Fredrickson, B. L. \& Cohn, M. A. (2008).Positive emotions. En M. Lewis, J. Haviland \& L. F. Barrett (Eds.), Handbook of emotions (3rd ed.). New York: GuilfordPress.

Hambleton, R. K. \& Patsula, L. (1999). Increasing the Validity of Adapted Tests: Myths to be Avoided and Guidelines for Improving Test Adaptation Practices. Journal of Applied Testing Technology, 1, 1-12.

Hervás, G. (2009). Psicologia positiva: Una introducción. Revista Interuniversitaria del Profesorado, 66, 2341.

Kaiser, H. F. (1970). A second generation Little Jiffy. Psychometrika, 35, 401-415. https://doi.org/10.1007/BF02291817

Kaplan, R. M. \& Saccuzzo, D. P. (2006). Pruebas psicológicas: principios, aplicaciones y temas (6ta ed). Madrid: International Thomson.

Kline, R. B. (1998). Principles and practice of structural equation modeling. New York, NY: Guilford Press.

Lloret-Segura, S., Ferreres-Traver, A., Hernández-Baeza, A., \& Tomás-Marco, I. (2014). El análisis factorial exploratorio de los ítems: una guía práctica, revisada y actualizada. Anales de Psicología, 30, 11511169. https://doi.org/10.6018/analesps.30.3.199361

Loewenthal, K. M. (2001). An introduction to psychological tests and scales (2da ed). Londres: Psychology Press.

Lucas, R. E., Diener, E. \& Larsen, R. J. (2003) Measuring positive emotions. En S. J. Lopez \& C. R. Snyder (Eds.), Positive psychological assessment: A handbook of models and measures. Washington: American Psychological Association. https://doi.org/10.1037/10612-000

Menghi, M. S., Rodriguez, L. M., Tortul, M. C., \& Moreno, J. E. (2017). Adaptación de la Escala de probabilidad de perdón a población adolescente argentina. Interdisciplinaria. Revista de Psicología y Ciencias Afines, 34(2), 489-498.

Norman, G. R. \& Streiner, D. L. (1996). Bioestadistica. Madrid: Mosby.

Pietro-Ursúa, M. \& Echegoyen, I. (2015). ¿Perdón a uno mismo, autoaceptación o restauración intrapersonal? Cuestiones abiertas en psicología del perdón. Papeles del psicólogo, 36(3), 230-237.

Regalia, C. \& Paleari, F. G. (2014). Forgiveness of Others Scale. Encyclopedia of Quality of Life and WellBeing Research, 2338-2340. https://doi.org/10.1007/978-94-007-0753-5_1077

Rodriguez, L. M., Oñate, M. E., \& Mesurado, B. (2017). Revisión del Cuestionario de Emociones Positivas para adolescentes. Propiedades psicométricas de la nueva versión abreviada. Universitas Psychologica, 16(3), 1-13. https://doi.org/10.11144/Javeriana.upsy16-3.rcep

Rosales Sarabia, R. M., Rivera Aragón. S., \& García Méndez, M. (2017). Revisión psicométrica de tres escalas del perdón: midiendola metamorfosis emocional. Acta de Investigación Psicológica, 7, 25772584. https://doi.org/10.1016/j.aipprr.2017.02.005

Rye, M. S., Loiacono, D. M., Folck, C. D., Olszewski, B. T., Heim, T. A., \& Madia, B. P. (2001). Evaluation of the psychometric properties of two forgiveness scales.Current Psychology: Developmental, Learning, Personality, Social, 20, 260-277. https://doi.org/10.1007/s12144-001-1011-6

Rye, M. S. (1998). Evaluation of a secular and a religiously integrated forgiveness group therapy program for college students who have been wronged by a romantic partner. Unpublished doctoral dissertation, Bowling Green State University, Bowling Green, OH.

Schmidt, C. M. (2008). Construcción de un cuestionario de emociones positivas en población entrerriana. Revista Iberoamericana de Evaluación Psicológica, 26(2), 117-139.

Worthington, E. L. \& Wade, N. (1999). The psychology or unforgiveness and forgiveness and implications for clinical practice. Journal of Social and Clinical Psychology, 18(4), 385-418. https://doi.org/10.1521/jscp.1999.18.4.385

Recibido 05-07-2017 | Aceptado 27-10-2017

Este trabajo se encuentra bajo una Licencia Creative Commons Atribución 4.0 Internacional que permite a terceros utilizar lo publicado siempre que se dé el crédito pertinente a los autores y a Psicodebate 